\title{
Collision times of random walks and applications to the Brownian web
}

\author{
David Coupier ${ }^{1}$, Kumarjit Saha ${ }^{2}$, Anish Sarkar ${ }^{3}$, and Viet Chi \\ $\operatorname{Tran}^{4}$ \\ ${ }^{1}$ Univ. de Valenciennes, CNRS \\ ${ }^{2}$ Ashoka University \\ ${ }^{3}$ Indian Statistical Institute, Delhi \\ ${ }^{4}$ Univ. Lille, CNRS
}

February 12, 2019

\begin{abstract}
Convergence of directed forests, spanning on random subsets of lattices or on point processes, towards the Brownian web has made the subject of an abundant literature, a large part of which relies on a criterion proposed by Fontes, Isopi, Newman and Ravishankar (2004). One of their convergence condition, called (B2), states that the probability of the event that there exists three distinct paths for a time interval of length $t(>0)$, all starting within a segment of length $\varepsilon$, is of small order of $\varepsilon$. This condition is often verified by applying an FKG type correlation inequality together with a coalescing time tail estimate for two paths. For many models where paths have complex interactions, it is hard to establish FKG type inequalities. In this article, we show that for a non-crossing path model, with certain assumptions, a suitable upper bound on expected first collision time among three paths can be obtained directly using Lyapunov functions. This, in turn, provides an alternate verification of Condition (B2). We further show that in case of independent simple symmetric one dimensional random walks or in case of independent Brownian motions, the expected value can be computed explicitly. We apply this alternate method of verification of (B2) to several models in the basin of attraction of the Brownian web studied earlier in the literature ([22, 14, 11]).
\end{abstract}

\section{Introduction}

The Brownian web can be described as a collection of coalescing one-dimensional Brownian motions starting from everywhere on a space-time plane $\mathbb{R}^{2}$. The origin of this random object goes back to the work of Arratia (see [1), where he studied the diffusive scaling limit of coalescing simple symmetric random walk paths starting from every point of $2 \mathbb{Z}$ at time 0 and showed that it converges to a collection of coalescing Brownian motions starting from every point of $\mathbb{R}$ at time 0 . The construction of a system of coalescing Brownian motions starting from 
every point in space and time was first given by Tóth and Werner in 26 where they used it to construct what they called "the true self-repelling motions". In [10], Fontes et al. finally introduced a suitable topology so that the Brownian web can be viewed as a random variable taking values in a Polish space. In particular, they developed a criterion for convergence of rescaled forests to the Brownian web, that we will recall later (Theorem 2.2.

The convergence conditions include two criteria about the number of distinct paths for a time interval of length $t(>0)$. In particular, their condition (B2) states that the probability of the event that there exists three distinct paths for a time interval of length $t(>0)$, all starting within a segment of length $\varepsilon$, is of small order of $\varepsilon$. For non-crossing path models, this condition is often verified by applying FKG type correlation inequality together with a bound on the distribution of the coalescence time between two paths. However, FKG is a strong property that is difficult to verify (even to expect) for many models, especially for models where paths have complex interactions.

The purpose of this article is to propose another method of verification of (B2) which is applicable for a non-crossing path models with certain assumptions. We show that under these assumptions, it is enough to have suitable estimate on the tail probability of the first collision time among three paths (see Proposition (3.2). Using homogeneity and Markovian properties of the model and using a Lyapunov function type technique, we prove a bound on the expected first collision time among three paths (Theorem 3.1). This allows us to use simple Markov inequality to obtain the tail estimate and verify condition (B2) without invoking FKG type inequalities.

Further, we show that part of this method, can be extended to explicitly calculate the expected first collision time among three paths in case of independent simple symmetric random walks and in case of independent Brownian motions (Theorem 3.3). Although, these results seem to be known, the only reference that we found was 24] [Lemma 12]. In Section 4]we apply our method to verify (B2) for two discrete drainage network models studied earlier in the literature: Scheidegger model [22] and Howard model [14]. Our domination condition (Condition (iii) of Proposition 2.3) is difficult to verify for forests in continuum spaces; however, it might be still possible to apply the core ideas of our method. As an example, the Poisson tree of Ferrari et al. [11, 12] is treated in Section 4.3 .

It should be mentioned here that there are other approaches for studying convergence to the Brownian web which do not require Conditon (B2) to check. Schertzer et. al. provided such an approach applicable for non-crossing path model (Theorem 6.6 of 23 ). Slightly stronger version of this approach appear in Theorem 26 of [7. Both these approaches applicable for non-crossing path models and do not require Markovian properties unless a suitable bound on the distribution of the coalescing time between two paths is available. Long before this, Newman et. al. developed an alternate condition replacing (B2) (Theorem 1.4 of [17]), which is applicable for crossing path models also. 


\section{Convergence to the Brownian web}

\subsection{The Brownian web}

We describe the Brownian web and the topology introduced in Fontes et al. [10], that we are going to work with. This subsection ends by recalling the convergence criterion proposed by these authors.

Let $\mathbb{R}_{c}^{2}$ denote the completion of the space time plane $\mathbb{R}^{2}$ with respect to the metric

$$
\rho\left(\left(x_{1}, t_{1}\right),\left(x_{2}, t_{2}\right)\right):=\left|\tanh \left(t_{1}\right)-\tanh \left(t_{2}\right)\right| \vee\left|\frac{\tanh \left(x_{1}\right)}{1+\left|t_{1}\right|}-\frac{\tanh \left(x_{2}\right)}{1+\left|t_{2}\right|}\right| .
$$

As a topological space $\mathbb{R}_{c}^{2}$ can be identified with the continuous image of $[-\infty, \infty]^{2}$ under a map that identifies the line $[-\infty, \infty] \times\{\infty\}$ with the point $(*, \infty)$, and the line $[-\infty, \infty] \times\{-\infty\}$ with the point $(*,-\infty)$. A path $\pi$ in $\mathbb{R}_{c}^{2}$ with starting time $\sigma_{\pi} \in[-\infty, \infty]$ is a mapping $\pi:\left[\sigma_{\pi}, \infty\right] \rightarrow[-\infty, \infty] \cup\{*\}$ such that $\pi(\infty)=*$ and, when $\sigma_{\pi}=-\infty, \pi(-\infty)=*$. Also $t \mapsto(\pi(t), t)$ is a continuous map from $\left[\sigma_{\pi}, \infty\right]$ to $\left(\mathbb{R}_{c}^{2}, \rho\right)$. We then define $\Pi$ to be the space of all paths in $\mathbb{R}_{c}^{2}$ with all possible starting times in $[-\infty, \infty]$.

The following metric, for $\pi_{1}, \pi_{2} \in \Pi$

$$
\begin{aligned}
d_{\Pi}\left(\pi_{1}, \pi_{2}\right):=\max \left\{\left|\tanh \left(\sigma_{\pi_{1}}\right)-\tanh \left(\sigma_{\pi_{2}}\right)\right|,\right. \\
\left.\sup _{t \geq \sigma_{\pi_{1}} \wedge \sigma_{\pi_{2}}}\left|\frac{\tanh \left(\pi_{1}\left(t \vee \sigma_{\pi_{1}}\right)\right)}{1+|t|}-\frac{\tanh \left(\pi_{2}\left(t \vee \sigma_{\pi_{2}}\right)\right)}{1+|t|}\right|\right\}
\end{aligned}
$$

makes $\Pi$ a complete, separable metric space. The metric $d_{\Pi}$ is slightly different from the original choice in [10] which is somewhat less natural as explained in [25]. Convergence in this metric can be described as locally uniform convergence of paths as well as convergence of starting times. In what follows, for $\mathbf{x}=$ $(\mathbf{x}(1), \mathbf{x}(2)) \in \mathbb{R}^{2}$ the notation $\pi^{\mathbf{x}}$ is used to denote a path starting from $\mathbf{x}$, i.e., $\pi^{\mathbf{x}}:[\mathbf{x}(2), \infty) \mapsto \mathbb{R}$.

Let $\mathcal{H}$ be the space of compact subsets of $\left(\Pi, d_{\Pi}\right)$ equipped with the Hausdorff metric $d_{\mathcal{H}}$ given by,

$$
d_{\mathcal{H}}\left(K_{1}, K_{2}\right):=\sup _{\pi_{1} \in K_{1}} \inf _{\pi_{2} \in K_{2}} d_{\Pi}\left(\pi_{1}, \pi_{2}\right) \vee \sup _{\pi_{2} \in K_{2}} \inf _{\pi_{1} \in K_{1}} d_{\Pi}\left(\pi_{1}, \pi_{2}\right) .
$$

Since $\left(\Pi, d_{\Pi}\right)$ is Polish, $\left(\mathcal{H}, d_{\mathcal{H}}\right)$ is also Polish. Let $B_{\mathcal{H}}$ be the Borel $\sigma$-algebra on the metric space $\left(\mathcal{H}, d_{\mathcal{H}}\right)$. In the following, for any $\mathbf{x}^{1}, \ldots, \mathbf{x}^{k} \in \mathbb{R}^{2}$, the notation $\left(W^{\mathbf{x}^{1}}, \ldots, W^{\mathbf{x}^{k}}\right)$ represents coalescing Brownian motions starting from $\mathbf{x}^{1}, \ldots, \mathbf{x}^{k}$ respectively. The Brownian web $\mathcal{W}$ is characterized as (Theorem 2.1 of [10]):

Theorem 2.1. There exists an $\left(\mathcal{H}, \mathcal{B}_{\mathcal{H}}\right)$ valued random variable $\mathcal{W}$ such that whose distribution is uniquely determined by the following properties:

(a) for each deterministic point $\mathbf{x} \in \mathbb{R}^{2}$ there is a unique path $\pi^{\mathbf{x}} \in \mathcal{W}$ almost surely;

(b) for a finite set of deterministic points $\mathbf{x}^{1}, \ldots, \mathbf{x}^{k} \in \mathbb{R}^{2}$, the collection $\left(\pi^{\mathbf{x}^{1}}, \ldots, \pi^{\mathbf{x}^{k}}\right)$ is distributed as $\left(W^{\mathbf{x}^{1}}, \ldots, W^{\mathbf{x}^{k}}\right)$; 
(c) for any countable deterministic dense set $\mathcal{D} \in \mathbb{R}^{2}, \mathcal{W}$ is the closure of $\left\{\pi^{\mathbf{x}}: \mathbf{x} \in \mathcal{D}\right\}$ in $\left(\Pi, d_{\Pi}\right)$ almost surely.

Note that the above theorem shows how to work with uncountably many Brownian motions as the collection is almost surely determined by only countably many coalescing Brownian motions.

\subsection{Convergence criterion of Fontes et al.}

Since the random variable $\mathcal{W}$ takes values in a Polish space, we can talk about weak convergence. Fontes et al. [10] provided the criteria to study weak convergence to the Brownian web $\mathcal{W}$. We present their convergence criteria for models with non-crossing paths only. A subset $\Gamma$ of $\Pi$ is said to be consists of non-crossing paths if there exist no $\pi_{1}, \pi_{2} \in \Gamma$ such that

$$
\left(\pi_{1}(t)-\pi_{2}(t)\right)\left(\pi_{1}(s)-\pi_{2}(s)\right)<0 \text { for some } t, s \geq \max \left\{\sigma_{\pi_{1}}, \sigma_{\pi_{2}}\right\}
$$

For a subset $\Gamma \subseteq \Pi$ of paths and for $t \in \mathbb{R}$ let $\Gamma^{t}:=\left\{\pi \in \Gamma: \sigma_{\pi} \leq t\right\}$ denote the set of paths which start 'before' time $t$. For $t>0$ and $t_{0}, a, b \in \mathbb{R}$ with $a<b$, we define a counting random variable as follows

$$
\eta_{\Gamma}\left(t_{0}, t ; a, b\right):=\#\left\{\pi\left(t_{0}+t\right): \pi \in \Gamma^{t_{0}} \text { and } \pi\left(t_{0}\right) \in[a, b]\right\} .
$$

The following theorem of [10] provides convergence criteria to the Brownian web for non-crossing path models.

Theorem 2.2. Let $\mathcal{X}_{n}, n \in \mathbb{N}$ be $\left(\mathcal{H}, B_{\mathcal{H}}\right)$ valued random variables with noncrossing paths. Assume that the following conditions hold:

$\left(I_{1}\right)$ Let $\mathcal{D}$ be a deterministic countable dense set of $\mathbb{R}^{2}$. For each $\mathbf{x} \in \mathcal{D}$, there exists $\pi_{n}^{\mathbf{x}} \in \mathcal{X}_{n}$ such that for any finite set of points $\mathbf{x}^{1}, \ldots, \mathbf{x}^{k} \in \mathcal{D}$, as $n \rightarrow$ $\infty$, we have $\left(\pi_{n}^{\mathbf{x}^{1}}, \ldots, \pi_{n}^{\mathbf{x}^{k}}\right)$ converges in distribution to $\left(W^{\mathbf{x}^{1}}, \ldots, W^{\mathbf{x}^{k}}\right)$.

$\left(B_{1}\right)$ For all $t>0$ and $a, t_{0} \in \mathbb{R}$,

$$
\limsup _{n \rightarrow \infty} \sup _{\left(a, t_{0}\right) \in \mathbb{R}^{2}} \mathbb{P}\left(\eta_{\mathcal{X}_{n}}\left(t_{0}, t ; a, a+\epsilon\right) \geq 2\right) \rightarrow 0 \text { as } \epsilon \downarrow 0 .
$$

$\left(B_{2}\right)$ For all $t>0$ and $a, t_{0} \in \mathbb{R}$,

$$
\frac{1}{\epsilon} \limsup _{n \rightarrow \infty} \sup _{\left(a, t_{0}\right) \in \mathbb{R}^{2}} \mathbb{P}\left(\eta_{\mathcal{X}_{n}}\left(t_{0}, t ; a, a+\epsilon\right) \geq 3\right) \rightarrow 0 \text { as } \epsilon \downarrow 0 .
$$

Then $\mathcal{X}_{n}$ converges in distribution to the standard Brownian web $\mathcal{W}$ as $n \rightarrow \infty$.

Fontes et al. 10 used this convergence criteria to prove that the collection of diffusively scaled coalescing one-dimensional simple symmetric random walk paths converges to the Brownian web. This model is described in more detail in Subsection 4.1. Since then, the convergence of coalescing path families (crossing as well as non-crossing) to the Brownian web has made the subject of an abundant literature $([3,5,6,6,[12,10,[17,20,[21,27])$. We refer to [23] for a review. 


\subsection{Main result: an alternative verification of (B2)}

As mentioned in the introduction, for non-crossing path models in order to verify (B2) it is enough to have sufficient tail decay for the first collision time among three paths and often it is verified using an FKG type correlation inequality. However, FKG property is difficult to verify for many models where paths have complex interactions, e.g., Howard's model [14, discrete directed spanning forest [20], directed spanning forest [2, 8] etc. Among these, for Howard's model, only a partial version of the FKG property is proved till now [5]. The main contribution of this paper is that, we propose an alternate method for verification of (B2) based on a bound for expected first collision time and show that this method is applicable for many non-crossing path models with certain Markovian properties and homogeneity assumptions (see Proposition 3.2 for detail).

To fix ideas let us introduce some notations. Suppose $\mathcal{V}$ is a locally finite random subset of $\mathbb{R}^{2}$ such that $\mathcal{V} \cap\{(x, s): x \in \mathbb{R}, s \geq t\}$ is nonempty for all $t \in \mathbb{R}$. Let $h: \mathbb{R}^{2} \rightarrow \mathcal{V}$ is a random map such that for each $(x, t) \in \mathbb{R}^{2}$, almost surely we have $h(x, t)(2)>t$ where $h(x, t)(i)$ denotes the $i$-th co-ordinate of $h(x, t)$ for $i=1,2$. The vertex $h(x, t)$ is the ancestor of $(x, t)$. We can define recursively the next ancestors, for $k \geq 1$, by $h^{k}(x, t):=h\left(h^{k-1}(x, t)\right)$, with $h^{0}(x, t)=(x, t)$. For each $(x, t) \in \mathbb{R}^{2}$, the path $\pi^{(x, t)}:[t, \infty) \rightarrow \mathbb{R}$ starting from $(x, t)$ is obtained by joining the successive vertices $h^{k-1}(x, t), h^{k}(x, t), k \geq 1$ linearly. It is useful to observe that both $h(x, t)$ and $\pi^{(x, t)}$ are defined for any $(x, t) \in \mathbb{R}^{2}$.

The collection of all the paths starting from the vertices in $\mathcal{V}$ is given as $\mathcal{X}:=\left\{\pi^{(x, t)}:(x, t) \in \mathcal{V}\right\}$. For a path $\pi \in \Pi$, for $n \geq 1$ and for normalization constants $\gamma, \sigma>0$, the $n$-th order diffusively scaled path is given by $\left\{\pi_{n}(t)=\right.$ $\left.\pi_{n}(\gamma, \sigma)(t)=\frac{1}{\sqrt{n} \sigma} \pi(n \gamma t)\right\}$. Corresponding collection of scaled paths is denoted by $\mathcal{X}_{n}:=\left\{\pi_{n}^{(x, t)}:(x, t) \in \mathcal{V}\right\}$. Let $\overline{\mathcal{X}}_{n}$ denote the closure of $\mathcal{X}_{n}$ in $\left(\Pi, d_{\Pi}\right)$. We assume that for each $n \geq 1, \overline{\mathcal{X}}_{n}$ is a $\left(\mathcal{H}, B_{\mathcal{H}}\right)$ valued random variable. Set $-\infty<x<y<z<\infty$ and consider the three paths $\pi^{(x, 0)}, \pi^{(y, 0)}, \pi^{(z, 0)}$ starting from the points $(x, 0),(y, 0)$ and $(z, 0)$ respectively. The first collision time of these three paths is defined as

$$
T_{(x, y, z)}:=\inf \left\{t \geq 0 \text { : either } \pi^{(y, 0)}(t)=\pi^{(x, 0)}(t) \text { or } \pi^{(z, 0)}(t)=\pi^{(y, 0)}(t)\right\} .
$$

The next proposition is motivated from the argument of Fontes et al. 12 and uses Markov inequality in place of FKG inequality.

Proposition 2.3. Suppose $(\mathcal{V}, h)$ satisfies the following conditions:

(i) [Non-crossing] For any $\left(x_{1}, t_{1}\right)$ and $\left(x_{2}, t_{2}\right)$ in $\mathbb{R}^{2}$, the paths $\pi^{\left(x_{1}, t_{1}\right)}, \pi^{\left(x_{2}, t_{2}\right)}$ are non-crossing.

(ii) [Homogeneity] For every $a, t_{0} \in \mathbb{R}$ and $t, \epsilon>0$, the distribution of the counting random variable $\eta_{\mathcal{X}_{n}}\left(t_{0}, t_{0}+t ; a, a+\epsilon\right)$ does not depend on $a, t_{0}$.

(iii) For each $t>0$, we have

$$
\begin{gathered}
\frac{1}{\epsilon} \limsup _{n \rightarrow \infty} \mathbb{P}\left(\left\{\pi(n t): \pi \in \mathcal{X}, \sigma_{\pi} \leq 0, \pi(0) \in[0, \epsilon \sqrt{n}]\right\}\right. \\
\left.\nsubseteq\left\{\pi^{(j, 0)}(n t): j \in[0,\lfloor\epsilon \sqrt{n}\rfloor+1] \cap \mathbb{Z}\right\}\right) \rightarrow 0
\end{gathered}
$$

as $\epsilon \downarrow 0$. 
(iv) For $x, y, z \in \mathbb{R}$ with $x<y<z$ and constants $C_{1}, C_{2}>0$ (independent of $x, y$ and $z)$, we have

$$
\mathbb{E}\left(T_{(x, y, z)}\right) \leq C_{1}+C_{2}(y-x)(z-y) .
$$

Then $\left\{\overline{\mathcal{X}}_{n}: n \geq 1\right\}$ satisfies $\left(B_{2}\right)$.

Proof. We prove the result for $\sigma=\gamma=1$, the general case being similar. From Condition (ii) [homogeneity], we observe that, for any $a, t_{0} \in \mathbb{R}$ and $t, \epsilon>0$,

$$
\mathbb{P}\left(\eta_{\mathcal{X}_{n}}\left(t_{0}, t_{0}+t ; a, a+\epsilon\right) \geq 3\right)=\mathbb{P}\left(\eta_{\mathcal{X}_{n}}(0, t ; 0, \epsilon) \geq 3\right) .
$$

Hence, it suffices to show that for any $t>0$,

$$
\frac{1}{\epsilon} \limsup _{n \rightarrow \infty} \mathbb{P}\left(\eta_{\mathcal{X}_{n}}(0, t ; 0, \epsilon) \geq 3\right)=\frac{1}{\epsilon} \limsup _{n \rightarrow \infty} \mathbb{P}\left(\eta_{\mathcal{X}}(0, n t ; 0, \epsilon \sqrt{n}) \geq 3\right) \rightarrow 0,
$$

as $\epsilon \downarrow 0$. Equation (5) means, for $\mathcal{X}$, that there exist three distinct paths starting between 0 and $\epsilon \sqrt{n}$ that do not coalesce before $n t$. We prove (5) for $t=1$. The argument for general $t>0$ is similar.

Let us define the set of paths starting from vertices $\{(j, 0): 0 \leq j \leq\lfloor\epsilon \sqrt{n}\rfloor+$ $1\}$ by $\mathcal{P}$. Let $B_{n}(\epsilon)$ denote the event $\left\{\left\{\pi(n): \pi \in \mathcal{X}_{1}, \sigma_{\pi} \leq 0, \pi(0) \in[0, \epsilon \sqrt{n}]\right\} \nsubseteq\right.$ $\{\pi(n): \pi \in \mathcal{P}\}\}$. On the complement of the event $B_{n}(\epsilon)$, there must be at least 3 distinct paths in $\mathcal{P}$ till time $n$, the event which we denote by $A_{\mathcal{P}}(3 ; n)$. Therefore, we have,

$$
\left\{\eta_{\mathcal{X}_{1}}(0, n ; 0, \epsilon \sqrt{n}) \geq 3\right\} \subseteq A_{\mathcal{P}}(3 ; n) \cup B_{n}(\epsilon) .
$$

Next, we estimate $\mathbb{P}\left(A_{\mathcal{P}}(3 ; n)\right)$. We follow a modification of the argument of Fontes et al. [12] and use the straightforward Markov inequality. For any $i \in \mathbb{Z}$ and $j \geq 2$, define the event where no coalescence occur before time $n$ between the paths starting from the vertices $(i, 0),(i+1,0)$ and $(i+j, 0)$, i.e.,

$$
E(i, j ; n):=\left\{\pi^{(i, 0)}(n)<\pi^{(i+1,0)}(n)<\pi^{(i+j, 0)}(n)\right\} .
$$

Note that the paths $\pi^{(i, 0)}, \pi^{(i+1,0)}$ and $\pi^{(i+j, 0)}$ are in $\mathcal{P}$ but need not be in $\mathcal{X}$. From non-crossing nature of paths (Condition (i)) it follows, if $A_{\mathcal{P}}(3 ; n)$ occurs then at least one of $E(i,\lfloor\epsilon \sqrt{n}\rfloor+1-i ; n)$ must occur for some $i=$ $0,1, \ldots,\lfloor\epsilon \sqrt{n}\rfloor-1$. Hence we have:

$$
A_{\mathcal{P}}(3 ; n) \subseteq \cup_{i=0}^{\lfloor\epsilon \sqrt{n}\rfloor-1} E(i,\lfloor\epsilon \sqrt{n}\rfloor+1-i ; n) .
$$

Using Condition (iv) and the Markov inequality,

$$
\begin{aligned}
& \mathbb{P}(E(i,\lfloor\epsilon \sqrt{n}\rfloor+1-i ; n))=\mathbb{P}\left(T_{(i, i+1,\lfloor\epsilon \sqrt{n}\rfloor+1)}>n\right) \\
& \leq \frac{1}{n} \mathbb{E}\left(T_{(i, i+1,\lfloor\epsilon \sqrt{n}\rfloor+1)}\right) \leq \frac{1}{n}\left[C_{1}+C_{2}(\lfloor\epsilon \sqrt{n}\rfloor-i)\right] \\
& \leq \frac{1}{n}\left[C_{1}+C_{2} \epsilon \sqrt{n}\right] .
\end{aligned}
$$


Thus, from (7),

$$
\begin{aligned}
& \limsup _{n \rightarrow \infty} \mathbb{P}\left(\eta_{\mathcal{X}_{n}}(0,1 ; 0, \epsilon) \geq 3\right) \\
& \leq \limsup _{n \rightarrow \infty}\left[\frac{1}{n} \sum_{i=0}^{\lfloor\epsilon \sqrt{n}\rfloor-1}\left[C_{1}+C_{2} \epsilon \sqrt{n}\right]+\mathbb{P}\left(B_{n}(\epsilon)\right)\right] \\
& \leq \limsup _{n \rightarrow \infty} \frac{1}{n}\left[C_{1} \epsilon \sqrt{n}+C_{2}(\epsilon \sqrt{n})^{2}\right]+\limsup _{n \rightarrow \infty} \mathbb{P}\left(B_{n}(\epsilon)\right) \\
& \leq C_{2} \epsilon^{2}+\limsup _{n \rightarrow \infty} \mathbb{P}\left(B_{n}(\epsilon)\right) .
\end{aligned}
$$

Hence, using condition (iii), $\frac{1}{\epsilon} \lim \sup _{n \rightarrow \infty} \mathbb{P}\left(\eta_{\mathcal{X}_{n}}(0,1 ; 0, \epsilon) \geq 3\right) \rightarrow 0$ as $\epsilon \downarrow$ 0 .

\section{First collision time of three paths}

Conditions (i), (ii) and (iii) of Propositions 2.3 are usually easy to read from the model. We focus in this section on Condition (iv). We first show that when the paths of $\mathcal{X}_{n}$ are non-crossing and exhibit certain Markov properties, then (4) can be obtained by Lyapunov techniques. This is done in Section 3.1. In case of coalescing simple symmetric one-dimensional random walks or in case of Brownian motions, we calculate the expectation of the first collision time explicitly. This is presented in Sections 3.2 and 3.3

\subsection{Control of the collision time expectation}

We start with a preliminary result on entrance time of a Markov chain with countable state space.

\subsubsection{An entrance time result for a Markov chain}

Let $\left\{Y_{j}: j \geq 0\right\}$ be such a Markov chain with countable state space $\mathcal{M}$. For any subset $M \subseteq \mathcal{M}$, let $\tau(M):=\inf \left\{n \geq 1: Y_{n} \in M\right\}$ be the first entrance time to $M$.

Theorem 3.1. Suppose there exist a function $V: \mathcal{M} \rightarrow[0, \infty), b \geq 0, p_{0}>0$ and two disjoint subsets $M_{0}, M_{1}$ of $\mathcal{M}$ such that

(i) $\mathbb{E}\left[V\left(Y_{1}\right)-V\left(Y_{0}\right) \mid Y_{0}=x\right] \leq-1+b \mathbb{I}\left\{x \in M_{1}\right\}$ for all $x \in M_{0}^{c}$;

(ii) $\mathbb{P}\left(Y_{1} \in M_{0} \mid Y_{0}=x\right) \geq p_{0}$ for all $x \in M_{1}$

where $\mathbb{I}(A)$ denote the indicator of the set $A$. Then for $x \in M_{0}^{c}$ we have

$$
\mathbb{E}\left[\tau\left(M_{0}\right) \mid Y_{0}=x\right] \leq V(x)+b / p_{0} .
$$

Note here that $M_{1} \cup M_{0}$ need not be finite. In our applications, we would have $V(x)=0$ for $x \in M_{0}$ and the class of states in $M_{0}$ is closed, i.e., if we enter this class, we would not get out of it.

Let us briefly explain the intuition behind this theorem. Condition (i) is essentially a Lyapunov type condition, where we have a strictly positive drift 
towards $M_{1} \cup M_{0}$, which by standard arguments would imply that the expected entrance time into $M_{1} \cup M_{0}$ for any $x \in\left(M_{1} \cup M_{0}\right)^{c}$ is bounded by constant multiple of $V(x)$. Condition (ii) ensures that each time the chain is in $M_{1}$, there is a strictly positive probability (bounded below) to hit $M_{0}$ at the next step and the chain should enter the set $M_{0}$ after a geometric number of time points. If $M_{1}$ is the empty set, the theorem is a known Lyapunov result. When $M_{1} \neq \emptyset$, this theorem shows that it is sufficient to focus on the entrance time to $M_{0} \cup M_{1}$.

Proof of Theorem 3.1. Let us define $W_{0}=0$ and for $n \geq 1$,

$$
W_{n}=V\left(Y_{n}\right)-V\left(Y_{0}\right)-\sum_{i=1}^{n} \mathbb{E}\left[V\left(Y_{i}\right)-V\left(Y_{i-1}\right) \mid Y_{i-1}\right] .
$$

Letting $\mathcal{F}_{n}=\sigma\left(Y_{0}, Y_{1}, \ldots, Y_{n}\right)$, it is easy to check that that $\left\{W_{n}: n \geq 0\right\}$ is $\mathcal{F}_{n}$-adapted martingale.

Let us set $Y_{0}=x \in M_{0}^{c}$ and $T_{0}:=\tau\left(M_{0}\right)$. Clearly $T_{0}$ is $\mathcal{F}_{n}$-measurable stopping time. Therefore, we have that $\left\{W_{n \wedge T_{0}}: n \geq 0\right\}$ is also $\mathcal{F}_{n}$-adapted martingale. Let $\mathbb{P}_{x}(\cdot):=\mathbb{P}\left(\cdot \mid Y_{0}=x\right)$ and $\mathbb{E}_{x}(\cdot):=\mathbb{E}\left(\cdot \mid Y_{0}=x\right)$ denote respectively the law and the expectation conditionally to the initial condition. Hence, we have $\mathbb{E}_{x}\left(W_{n \wedge T_{0}}\right)=\mathbb{E}_{x}\left(W_{0 \wedge T_{0}}\right)=0$ for all $n \geq 1$. Thus, using condition (i), we obtain that

$$
\begin{aligned}
\mathbb{E}_{x}\left[V\left(Y_{n \wedge T_{0}}\right)\right] & =V(x)+\mathbb{E}_{x}\left[\sum_{i=1}^{n \wedge T_{0}} \mathbb{E}_{x}\left[V\left(Y_{i}\right)-V\left(Y_{i-1}\right) \mid Y_{i-1}\right]\right] \\
& \leq V(x)+\mathbb{E}_{x}\left[\sum_{i=1}^{n \wedge T_{0}}-1+b \mathbb{I}\left\{Y_{i-1} \in M_{1}\right\}\right] \\
& =V(x)-\mathbb{E}_{x}\left(n \wedge T_{0}\right)+b \mathbb{E}_{x}\left[\sum_{i=1}^{n \wedge T_{0}} \mathbb{I}\left\{Y_{i-1} \in M_{1}\right\}\right]
\end{aligned}
$$

since for any $n \geq 1$ and $i=0,1, \ldots, n \wedge T_{0}-1, Y_{i} \in M_{0}^{c}$. Hence, transferring $\mathbb{E}_{x}\left(n \wedge T_{0}\right)$ to the other side and observing that $V$ is a non-negative function, we have

$$
\mathbb{E}_{x}\left(n \wedge T_{0}\right) \leq \mathbb{E}_{x}\left(n \wedge T_{0}\right)+\mathbb{E}_{x}\left[V\left(Y_{n \wedge T_{0}}\right)\right] \leq V(x)+b \mathbb{E}_{x}\left[\sum_{i=1}^{n \wedge T_{0}} \mathbb{I}\left\{Y_{i-1} \in M_{1}\right\}\right]
$$

We argue below that $T_{0}<\infty$ almost surely and $\sum_{i=1}^{n \wedge T_{0}} \mathbb{I}\left\{Y_{i-1} \in M_{1}\right\}$ is stochastically dominated by a geometric random variable (total number of trials before a success) with success probability $p_{0}$. Hence applying MCT on the left hand side of above equation, we obtain the required result.

Let $T_{1}:=\tau\left(M_{0} \cup M_{1}\right)$. From Theorem 11.3.4 in Meyn and Tweedie [15], assumption (i) implies that for all $x \notin M_{0} \cup M_{1}, \mathbb{E}_{x}\left(T_{1}\right) \leq V(x)$ so that $T_{1}<\infty$ almost surely. Now, for $x \in\left(M_{0} \cup M_{1}\right)^{c}$, the chain will almost surely enter the set $M_{0} \cup M_{1}$ in finite time and when it enters $M_{0} \cup M_{1}$, if it hits the set $M_{0}$ we are done. If it hits the set $M_{1}$, it has a strictly positive probability $p_{0}$ of hitting the set $M_{0}$ at the next step. So, in finitely many steps, the chain will either hit $M_{0}$ or will go out to $\left(M_{0} \cup M_{1}\right)^{c}$. Again, if the chain hits $M_{0}$, we are done. If the chain is in $\left(M_{0} \cup M_{1}\right)^{c}$, it is back at the starting situation. So, again it will 
hit the set $M_{0} \cup M_{1}$ in finite time and so on. Since $p_{0}>0$, the chain can only go out of $M_{1}$ to $\left(M_{0} \cup M_{1}\right)^{c}$ finitely many times, before it hits the set $M_{0}$. Thus, the chain will hit the set $M_{0}$ in finite time almost surely. When the chain starts from $M_{1}$, the situation is as above without first having to hit the set $M_{0} \cup M_{1}$. So, all cases, $T_{0}<\infty$ almost surely.

For every time point $i<n \wedge T_{0}$, the chain spends in $M_{1}$ it has a probability of at least $p_{0}$ of hitting the set $M_{0}$. Thus, taking hitting $M_{0}$ at the next time point as success, it is like conducting a geometric trial with success probability at least $p_{0}$ and hence the number of time points $i<n \wedge T_{0}$ is stochastically dominated by a geometric random variable with success probability $p_{0}$. For a formal proof, we will modify the chain's behaviour by making each state in $M_{0}$ an absorbing state. Since we are only interested in time points before the chain visits $M_{0}$, the distribution of $\sum_{i=1}^{n \wedge T_{0}} \mathbb{I}\left\{Y_{i-1} \in M_{1}\right\}$ is unaffected by this modification. With a little abuse of notation, we will continue to denote the modified chain by $Y_{n}$. Next define $\sigma_{0}=0$ and for $i \geq 1, \sigma_{i}=\inf \left\{n>\sigma_{i-1}: Y_{i} \in M_{1}\right\}$. Here we follow the convention that the infimum of an empty set $+\infty$. Observe that $\sigma_{i}$ is a $\mathcal{F}_{n}$ stopping time. Let $N=\sup \left\{k: \sigma_{k}<\infty\right\}$. We also observe that

$$
\sum_{i=1}^{n \wedge T_{0}} \mathbb{I}\left\{Y_{i-1} \in M_{1}\right\} \leq \sum_{i=1}^{\infty} \mathbb{I}\left\{Y_{i-1} \in M_{1}\right\}=N
$$

Now, using the fact that each state in $M_{0}$ is absorbing, we have for $k \geq 1$,

$$
\begin{aligned}
\mathbb{P}_{x}(N>k) & =\mathbb{P}_{x}\left(\sigma_{k+1}<\infty\right) \\
& =\mathbb{P}_{x}\left(Y_{\sigma_{i}} \in M_{1}, Y_{\sigma_{i}+1} \in M_{0}^{c} \text { for } 1 \leq i \leq k, \sigma_{k+1}<\infty\right) \\
& \leq \mathbb{P}_{x}\left(Y_{\sigma_{i}} \in M_{1}, Y_{\sigma_{i}+1} \in M_{0}^{c} \text { for } 1 \leq i \leq k\right) \\
& =\mathbb{E}_{x}\left[\mathbb{E}\left(\prod_{i=1}^{k} \mathbb{I}\left(Y_{\sigma_{i}} \in M_{1}, Y_{\sigma_{i}+1} \in M_{0}^{c}\right) \mid \mathcal{F}_{\sigma_{k}}\right)\right] \\
& =\mathbb{E}_{x}\left[\prod_{i=1}^{k-1} \mathbb{I}\left(Y_{\sigma_{i}} \in M_{1}, Y_{\sigma_{i}+1} \in M_{0}^{c}\right) \mathbb{I}\left(Y_{\sigma_{k}} \in M_{1}\right) \mathbb{P}_{Y_{\sigma_{k}}}\left(Y_{1} \in M_{0}^{c}\right)\right] \\
& \leq\left(1-p_{0}\right) \mathbb{E}_{x}\left[\prod_{i=1}^{k-1} \mathbb{I}\left(Y_{\sigma_{i}} \in M_{1}, Y_{\sigma_{i}+1} \in M_{0}^{c}\right)\right] \\
& \leq\left(1-p_{0}\right)^{k}
\end{aligned}
$$

following the above conditioning argument step by step. This proves the stochastic domination.

\subsubsection{Application to the expectation of first collision times}

Let us construct a discrete drainage network model where the vertex set $\mathcal{V}$ is a random subset of $\mathbb{Z}^{2}$ satisfying some homogeneity and Markovian properties (as mentioned in Proposition 3.2) and apply Theorem 3.1 to show that the Condition (iii) of Proposition 2.3 holds. We assume that for any $(x, t) \in \mathbb{Z}^{2}$, it's ancestor $h(x, t)$ sits on the next level, i.e., $h(x, t)(2)=t+1$ where $h(x, t)(i)$ is the $i$-th co-ordinate of $h(x, t)$ for $i=1,2$. As in Proposition 2.3, $\mathcal{X}$ denotes the collection of all paths starting from $\mathcal{V}$ and $\mathcal{X}_{n}$ is the corresponding collection 
of $n$-th order diffusively scaled paths. Define $\mathcal{S}=\left\{(u, v) \in \mathbb{Z}^{2}: u, v \geq 0\right\}$ and $\mathcal{S}_{0}=\{(u, v) \in \mathcal{S}: u v=0\} . \mathcal{S}\left(\right.$ resp. $\left.\mathcal{S}_{0}\right)$ plays the role of $\mathcal{M}$ (resp. $\left.\mathcal{M}_{0}\right)$ in Theorem [3.1. We are now ready to state our Proposition regarding alternate verification of (B2).

Proposition 3.2. Suppose $(\mathcal{V}, h)$ satisfies the following conditions:

(i) [Markov property] For $x, y, z \in \mathbb{Z}$ with $x<y<z$, the process $\left\{X_{j}:=\right.$ $\left.\left(\pi^{(y, 0)}(j)-\pi^{(x, 0)}(j), \pi^{(z, 0)}(j)-\pi^{(y, 0)}(j)\right): j \geq 0\right\}$ forms a Markov chain with state space $\mathcal{S}$.

(ii) [Control of the collision time] There exist a subset $\mathcal{S}_{1}$ of $\mathcal{S} \backslash \mathcal{S}_{0}$ and $p_{0}>0$, such that

(a) there exist positive constants $d_{1}$ and $d_{2}$ such that for all $(l, m) \notin \mathcal{S}_{0}$,

$$
\begin{aligned}
& \mathbb{E}\left(X_{1}(1) X_{1}(2)-X_{0}(1) X_{0}(2) \mid X_{0}=(l, m)\right) \\
& \quad \leq-d_{1}+d_{2} \mathbf{1}_{\left\{(l, m) \in \mathcal{S}_{1}\right\}}
\end{aligned}
$$

where $X_{n}=\left(X_{n}(1), X_{n}(2)\right)$.

(b) For all $(l, m) \in \mathcal{S}_{1}, \mathbb{P}\left(X_{1} \in \mathcal{S}_{0} \mid X_{0}=(l, m)\right) \geq p_{0}$.

Then for any $-\infty<x<y<z<\infty$, there exist positive constants $C_{1}, C_{2}$ (independent of $x, y, z)$ such that

$$
\mathbb{E}\left(T_{(x, y, z)}\right) \leq C_{1}+C_{2}(y-x)(z-y) .
$$

In addition, if $(\mathcal{V}, h)$ satisfies Condtion (ii) of Proposition 2.3, then $\left\{\overline{\mathcal{X}}_{n}: n \geq\right.$ 1) satisfies (B2).

Note that we have considered the state space of the Markov chain as the first quadrant, which implies that the paths are automatically non-crossing. Since the vertex set $\mathcal{V}$ is a random subset of $\mathbb{Z}^{2}$, and for any $(x, t) \in \mathbb{Z}^{2}$ we have $h(x, t)(2)=t+1$ almost surely, hence Condition (iii) of Proposition 2.3 automatically holds.

Proof. Consider the function $V: \mathcal{S} \rightarrow[0, \infty)$ defined by

$$
V(u, v)=\frac{u v}{d_{1}} .
$$

Part (a) and part (b) of Assumption (ii) of Proposition 3.2 ensure that Conditions (i) and (ii) of Theorem 3.1 hold for the markov chain $\left\{X_{j}: j \geq 0\right\}$.

For any $-\infty<x<y<z<\infty$, first collision time for the paths $\pi^{(x, 0)}, \pi^{(y, 0)}$ and $\pi^{(z, 0)}$ is same as the entrance time to the set $\mathcal{S}_{0}$ for the Markov chain $\left\{X_{j}: j \geq 0\right\}$. Thus, the bound for $\mathbb{E}\left(T_{(x, y, z)}\right)$ follows from Theorem 3.1. Finally the proof follows from Proposition 2.3.

\subsection{First collision time of three random walks}

The calculations leading to equation (10) yields a surprising exact computational result for collision time among three simple symmetric random walks. With $V(\cdot)$ being defined as the product of the distances between the pair of the left and 
the middle random walk and the pair of middle and right random walk (see below for exact definition), it turns out that the condition (i) in Theorem 3.1 is an equality with $M_{1}$ being the empty set. Though the exact computation is not required for the convergence to the Brownian web, we give it here for the sake of completeness.

Let us consider three symmetric nearest neighbour independent random walks, which we denote by $S_{n}^{(L)}, S_{n}^{(M)}$ and $S_{n}^{(R)}$, starting from $-2 i, 0$ and $2 j$ respectively with $i, j \geq 1$. Given three independent sequences of i.i.d increment random variables $\left\{I_{k}^{(L)}: k \geq 1\right\},\left\{I_{k}^{(M)}: k \geq 1\right\}$ and $\left\{I_{k}^{(R)}: k \geq 1\right\}$ with Rademacher distributions (each of these random variables take values +1 and -1 with probability $1 / 2$ ), the random walks are represented by

$$
S_{n}^{(L)}=-2 i+\sum_{k=1}^{n} I_{k}^{(L)}, \quad S_{n}^{(M)}=\sum_{k=1}^{n} I_{k}^{(M)} \quad \text { and } \quad S_{n}^{(R)}=2 j+\sum_{k=1}^{n} I_{k}^{(R)} .
$$

Let us define the associated filtration $\left\{\mathcal{F}_{n}: n \geq 0\right\}$ where $\mathcal{F}_{n}=\sigma\left(I_{k}^{(L)}, I_{k}^{(M)}, I_{k}^{(R)}\right.$ : $k \leq n)$ is the $\sigma$-algebra generated by the increment random variables up to time $n$.

We define the meeting times of these random walks by

$$
\begin{aligned}
\tau_{(L, M)} & :=\inf \left\{n \geq 1: S_{n}^{(L)}=S_{n}^{(M)}\right\} \quad \text { and } \\
\tau_{(M, R)} & :=\inf \left\{n \geq 1: S_{n}^{(M)}=S_{n}^{(R)}\right\} .
\end{aligned}
$$

We know that $\tau_{(L, M)}$ and $\tau_{(M, R)}$ are almost surely finite, but that their expectations are infinite. Let us define the first collision time of these random walks by

$$
\tau_{C}:=\min \left\{\tau_{(L, M)}, \tau_{(M, R)}\right\} .
$$

Theorem 3.3. With the notation as above, we have $\mathbb{E}\left(\tau_{C}\right)=4 i j$.

Although this theorem seems to be known, the only reference that we found was [24, Lemma 12]. We give the proof of the theorem for the sake of completeness. Before we proceed further, let us set up the notation. For all $n \geq 0$, we denote the distances between the paths by

$$
D_{n}^{(L, M)}=S_{n}^{(M)}-S_{n}^{(L)} \quad \text { and } \quad D_{n}^{(M, R)}=S_{n}^{(R)}-S_{n}^{(M)} .
$$

We have $D_{0}^{(L, M)}=2 i$ and $D_{0}^{(M, R)}=2 j$. Also set the maximum distance between the pairs as

$$
M_{n}=\max \left\{D_{n}^{(L, M)}, D_{n}^{(M, R)}\right\} .
$$

Remark 3.4. It is obvious that at the first collision time, only one pair of random walks can meet. Assuming Theorem [3.3, we can also compute that the other pair of paths is at an expected distance of $2(i+j)$ at the collision time. Since at $\tau_{C}$, at least one of $D_{\tau_{C}}^{(L, M)}$ or $D_{\tau_{C}}^{(M, R)}$ is zero, we have $M_{\tau_{C}}=$ $D_{\tau_{C}}^{(L, M)}+D_{\tau_{C}}^{(M, R)}$. But we may write

$$
D_{n}^{(L, M)}+D_{n}^{(M, R)}-2(i+j)=\sum_{j=1}^{n}\left(I_{j}^{(R)}-I_{j}^{(L)}\right) .
$$


Therefore, using Wald's identity, we have

$$
\mathbb{E}\left(M_{\tau_{C}}-2(i+j)\right)=\mathbb{E}\left(D_{\tau_{C}}^{(L, M)}+D_{\tau_{C}}^{(M, R)}-2(i+j)\right)=0 .
$$

Thus, $\mathbb{E}\left(M_{\tau_{C}}\right)=2(i+j)$.

The main observation is that we may rewrite $\tau_{C}$ as

$$
\tau_{C}=\inf \left\{n \geq 1: D_{n}^{(L, M)} D_{n}^{(M, R)}=0\right\} .
$$

Next we have two straightforward Propositions which involve the product of the distances.

Proposition 3.5. The family $\left\{D_{n}^{(L, M)} D_{n}^{(M, R)}+n: n \geq 0\right\}$ is an $\mathcal{F}_{n}$-adapted martingale.

Proposition 3.6. The family $\left\{D_{n}^{(L, M)} D_{n}^{(M, R)}\left(D_{n}^{(L, M)}+D_{n}^{(M, R)}\right): n \geq 0\right\}$ is an $\mathcal{F}_{n}$-adapted martingale.

Assuming the Propositions, we can now prove Theorem 3.3

Proof of Theorem 3.3. By Proposition 3.5 we have that, $\left\{D_{n \wedge \tau_{C}}^{(L, M)} D_{n \wedge \tau_{C}}^{(M, R)}+n \wedge\right.$ $\left.\tau_{C}: n \geq 0\right\}$ is a martingale and hence for any $n \geq 1$,

$$
\begin{aligned}
\mathbb{E}\left(D_{n \wedge \tau_{C}}^{(L, M)} D_{n \wedge \tau_{C}}^{(M, R)}+n \wedge \tau_{C}\right) & =\mathbb{E}\left(D_{0 \wedge \tau_{C}}^{(L, M)} D_{0 \wedge \tau_{C}}^{(M, R)}+0 \wedge \tau_{C}\right) \\
& =\mathbb{E}\left(D_{0}^{(L, M)} D_{0}^{(M, R)}\right)=4 i j .
\end{aligned}
$$

This is the version of equation (10) in this case. Since $\tau_{(L, M)}, \tau_{(M, R)}<+\infty$ almost surely, we have that $\tau_{C}<+\infty$ almost surely. By the monotone convergence theorem, we have that, as $n \rightarrow \infty, \mathbb{E}\left(n \wedge \tau_{C}\right) \rightarrow \mathbb{E}\left(\tau_{C}\right)$. Since $\tau_{C}<+\infty$ almost surely, we have that, almost surely, as $n \rightarrow \infty, D_{n \wedge \tau_{C}}^{(L, M)} D_{n \wedge \tau_{C}}^{(M, R)} \rightarrow$ $D_{\tau_{C}}^{(L, M)} D_{\tau_{C}}^{(M, R)}=0$. To complete the proof, we show that, as $n \rightarrow \infty$,

$$
\mathbb{E}\left(D_{n \wedge \tau_{C}}^{(L, M)} D_{n \wedge \tau_{C}}^{(M, R)}\right) \rightarrow 0 .
$$

From Proposition 3.6 we have that $\left\{D_{n \wedge \tau_{C}}^{(L, M)} D_{n \wedge \tau_{C}}^{(M, R)}\left(D_{n \wedge \tau_{C}}^{(L, M)}+D_{n \wedge \tau_{C}}^{(M, R)}\right): n \geq\right.$ $0\}$ is also a martingale and hence, for any $n \geq 1$,

$$
\begin{aligned}
& \mathbb{E}\left[D_{n \wedge \tau_{C}}^{(L, M)} D_{n \wedge \tau_{C}}^{(M, R)}\left(D_{n \wedge \tau_{C}}^{(L, M)}+D_{n \wedge \tau_{C}}^{(M, R)}\right)\right] \\
& =\mathbb{E}\left[D_{0 \wedge \tau_{C}}^{(L, M)} D_{0 \wedge \tau_{C}}^{(M, R)}\left(D_{0 \wedge \tau_{C}}^{(L, M)}+D_{0 \wedge \tau_{C}}^{(M, R)}\right)\right] \\
& =\mathbb{E}\left[D_{0}^{(L, M)} D_{0}^{(M, R)}\left(D_{0}^{(L, M)}+D_{0}^{(M, R)}\right)\right]=8 i j(i+j) .
\end{aligned}
$$

Therefore, observing that $D_{n \wedge \tau_{C}}^{(L, M)}$ and $D_{n \wedge \tau_{C}}^{(M, R)}$ are both non-negative, we have

$$
\begin{aligned}
& \sup _{n \geq 1} \mathbb{E}\left[\left(D_{n \wedge \tau_{C}}^{(L, M)} D_{n \wedge \tau_{C}}^{(M, R)}\right)^{3 / 2}\right] \\
& \leq \frac{1}{2} \sup _{n \geq 1} \mathbb{E}\left[D_{n \wedge \tau_{C}}^{(L, M)} D_{n \wedge \tau_{C}}^{(M, R)}\left(D_{n \wedge \tau_{C}}^{(L, M)}+D_{n \wedge \tau_{C}}^{(M, R)}\right)\right]=4 i j(i+j)
\end{aligned}
$$

and hence $\left\{D_{n \wedge \tau_{C}}^{(L, M)} D_{n \wedge \tau_{C}}^{(M, R)}: n \geq 1\right\}$ is a sequence of uniformly integrable random variables. We conclude (16) using Theorem 16.13. of Billingsley [4. 
Next we prove the Propositions which are straightforward.

Proof of Proposition 3.5. This proposition follows from straightforward calculation:

$$
\begin{aligned}
& \mathbb{E}\left[\left(D_{n+1}^{(L, M)} D_{n+1}^{(M, R)}+n+1\right)-\left(D_{n}^{(L, M)} D_{n}^{(M, R)}+n\right) \mid \mathcal{F}_{n}\right] \\
& =D_{n}^{(L, M)} \mathbb{E}\left[\left(I_{n+1}^{(R)}-I_{n+1}^{(M)}\right) \mid \mathcal{F}_{n}\right]+D_{n}^{(M, R)} \mathbb{E}\left[\left(I_{n+1}^{(M)}-I_{n+1}^{(L)}\right) \mid \mathcal{F}_{n}\right] \\
& \quad+\mathbb{E}\left[\left(I_{n+1}^{(M)}-I_{n+1}^{(L)}\right)\left(I_{n+1}^{(R)}-I_{n+1}^{(M)}\right) \mid \mathcal{F}_{n}\right]+1 \\
& \left.=\mathbb{E}\left[I_{n+1}^{(M)} I_{n+1}^{(R)}-\left(I_{n+1}^{(M)}\right)^{2}-I_{n+1}^{(L)} I_{n+1}^{(R)}+I_{n+1}^{(L)} I_{n+1}^{(M)}\right)\right]+1=0 .
\end{aligned}
$$

Proof of Proposition 3.6. Again straightforward calculations yield the result. We have

$$
\begin{aligned}
& D_{n+1}^{(L, M)} D_{n+1}^{(M, R)}\left(D_{n+1}^{(L, M)}+D_{n+1}^{(M, R)}\right)-D_{n}^{(L, M)} D_{n}^{(M, R)}\left(D_{n}^{(L, M)}+D_{n}^{(M, R)}\right) \\
& =D_{n}^{(L, M)} D_{n}^{(M, R)}\left(I_{n+1}^{(R)}-I_{n+1}^{(L)}\right)+D_{n}^{(L, M)}\left(D_{n}^{(L, M)}+D_{n}^{(M, R)}\right)\left(I_{n+1}^{(R)}-I_{n+1}^{(M)}\right) \\
& \quad+D_{n}^{(L, M)}\left(I_{n+1}^{(R)}-I_{n+1}^{(M)}\right)\left(I_{n+1}^{(R)}-I_{n+1}^{(L)}\right) \\
& \quad+D_{n}^{(M, R)}\left(D_{n}^{(L, M)}+D_{n}^{(M, R)}\right)\left(I_{n+1}^{(M)}-I_{n+1}^{(L)}\right) \\
& \quad+D_{n}^{(M, R)}\left(I_{n+1}^{(M)}-I_{n+1}^{(L)}\right)\left(I_{n+1}^{(R)}-I_{n+1}^{(L)}\right) \\
& \quad+\left(D_{n}^{(L, M)}+D_{n}^{(M, R)}\right)\left(I_{n+1}^{(M)}-I_{n+1}^{(L)}\right)\left(I_{n+1}^{(R)}-I_{n+1}^{(M)}\right) \\
& \quad+\left(I_{n+1}^{(M)}-I_{n+1}^{(L)}\right)\left(I_{n+1}^{(R)}-I_{n+1}^{(M)}\right)\left(I_{n+1}^{(R)}-I_{n+1}^{(L)}\right) .
\end{aligned}
$$

The conditional expectation with respect to $\mathcal{F}_{n}$ of the first term, second term and the fourth term is clearly 0 . We observe that that the conditional expectation with respect to $\mathcal{F}_{n}$ of the third term is $D_{n}^{(L, M)}$, the fifth term is $D_{n}^{(M, R)}$ and the sixth term is $-\left(D_{n}^{(L, M)}+D_{n}^{(M, R)}\right)$. The last term is independent of $\mathcal{F}_{n}$ and has expectation 0 . This proves the result.

\subsection{First collision time of three Brownian motions}

As one can expect, these results generalize to the Brownian motion set up in a straightforward way. Let us start with three independent Brownian motions $\left\{\left(B_{t}^{(L)}, B_{t}^{(M)}, B_{t}^{(R)}\right): t \geq 0\right\}$ started from $-x, 0$ and $y$ respectively, for $x, y>0$. We also define the associated filtration $\left\{\mathcal{F}_{t}: t \geq 0\right\}$ where $\mathcal{F}_{t}=\sigma\left(B_{s}^{(L)}, B_{s}^{(M)}, B_{s}^{(R)}: s \leq t\right)$.

Again, let us define

$$
\tau_{C}^{(B)}=\inf \left\{t>0:\left(B_{t}^{(M)}-B_{t}^{(L)}\right)\left(B_{t}^{(R)}-B_{t}^{(M)}\right)=0\right\} .
$$

Then, we have the following result

Theorem 3.7. With the notation as above, we have $\mathbb{E}\left(\tau_{C}^{(B)}\right)=x y$.

The proof follows from the propositions below which are exact replica of Propositions 3.5 and 3.6. exactly as in the discrete case. 
Proposition 3.8. We have $\left\{\left(B_{t}^{(M)}-B_{t}^{(L)}\right)\left(B_{t}^{(R)}-B_{t}^{(M)}\right)+t: t \geq 0\right\}$ is a $\left(\mathcal{F}_{t}\right)$-adapted martingale.

Proposition 3.9. We have $\left\{\left(B_{t}^{(M)}-B_{t}^{(L)}\right)\left(B_{t}^{(R)}-B_{t}^{(M)}\right)\left(B_{t}^{(R)}-B_{t}^{(L)}\right): t \geq 0\right\}$ is a $\left(\mathcal{F}_{t}\right)$-adapted martingale.

Remark 3.10. It should be observed that there is nothing surprising in the above Propositions as well as Propositions 3.5 and 3.6. For example, we can write

$$
\begin{aligned}
& \left(B_{t}^{(M)}-B_{t}^{(L)}\right)\left(B_{t}^{(R)}-B_{t}^{(M)}\right)+t \\
& =B_{t}^{(M)} B_{t}^{(R)}-B_{t}^{(L)} B_{t}^{(R)}+B_{t}^{(L)} B_{t}^{(M)}-\left[\left(B_{t}^{(M)}\right)^{2}-t\right]
\end{aligned}
$$

Since Brownian motions are themselves martingales and since products of independent martingales and sums of martingales are martingales, it is easy to observe that the first three terms are martingales. Since $t$ is the quadratic variation process of the Brownian motion, the last term is also a martingale. With these arguments, other processes can also be shown to be martingales.

\section{Applications to drainage network models}

We now apply Propositions 2.3 and 3.2 to some directed forests of the literature and provide an alternate verification of $\left(B_{2}\right)$ for these models.

\subsection{Scheidegger's model}

We start with the system of coalescing simple symmetric random walks starting from every point on $\mathbb{Z}_{\text {even }}^{2}:=\{(x, t): x+t$ even $\}$.

Let $\left\{b_{(x, t)}:(x, t) \in \mathbb{Z}_{\text {even }}^{2}\right\}$ be an i.i.d. collection such that

$$
b_{(x, t)}= \begin{cases}+1 & \text { with probability } 1 / 2 \\ -1 & \text { with probability } 1 / 2\end{cases}
$$

Define $h: \mathbb{Z}^{2} \mapsto \mathbb{Z}_{\text {even }}^{2}$ as

$$
h(x, t)= \begin{cases}\left(x+b_{(x, t)}, t+1\right) & \text { if }(x, t) \in \mathbb{Z}_{\text {even }}^{2} \\ (x, t+1) & \text { otherwise } .\end{cases}
$$

For $k \geq 1$ let $h^{k}(x, t):=h\left(h^{k-1}(x, t)\right)$ with $h^{0}(x, t)=(x, t)$. For $(x, t) \in \mathbb{Z}^{2}$ let $\pi^{(x, t)}:[t, \infty) \rightarrow \mathbb{R}$ be the path starting at $(x, t)$ obtained by joining the successive vertices $h^{k-1}(x, t), h^{k}(x, t), k \geq 1$ by straight line segments. Let $\mathcal{X}:=\left\{\pi^{(x, t)}:(x, t) \in \mathbb{Z}_{\text {even }}^{2}\right\}$, be the collection of all paths starting from $\mathbb{Z}_{\text {even }}^{2}$. For $n \geq 1$ and for normalization constants $\gamma, \sigma>0$, with slight abuse of notation let $\mathcal{X}_{n}=\mathcal{X}_{n}(\gamma, \sigma)$ denote the collection of the scaled paths. For each $n \geq 1$, $\overline{\mathcal{X}}_{n}$, the closure of $\mathcal{X}_{n}$ in $\left(\Pi, d_{\Pi}\right)$, is a $\left(\mathcal{H}, B_{\mathcal{H}}\right)$ valued random variable.

Fontes et. al 10] proved that $\overline{\mathcal{X}}_{n}$ converges in distribution to the Brownian web with $\gamma=\sigma=1$ (see Theorem 6.1 of Fontes et al. [10]). To verify the Condition $\left(B_{2}\right)$, they prove a version of FKG inequality for paths and using it, along with the coalescing time tail estimate for a pair of random walks, to 
derive suitable bounds. In this case it is not hard to obtain FKG inequalities as the paths are independent till the time of coalescence. On the other hand, we proved in Section 3 that the expected first collision time of three simple symmetric random walks starting at $2 i$ and $2 j$ is exactly $4 i j$, which gives the same order bound (with known constant values) as obtained by Fontes et al. However, as a warmup, we show that Proposition 3.2 is applicable here. Note that the exact value of this expectation is not required for our calculations.

In this case, we have $\mathcal{V}=\mathbb{Z}_{\text {even }}^{2}$ and $h(\cdot)$ is defined as above. From the construction it is clear that the paths are non-crossing and Condition (i) of Proposition 2.3 is satisfied. Because of the i.i.d. nature of the collection of random variables $\left\{b_{(x, t)}:(x, t) \in \mathbb{Z}_{\text {even }}^{2}\right\}$, the homogeneity condition, i.e., Condition (ii) of Proposition 2.3 is also satisfied.

For any $(x, 0) \in \mathbb{Z}_{\text {even }}^{2}, h^{j+1}(x, 0)(1)=h^{j}(x, 0)(1)+b_{\left(h^{j}(x, 0)\right)}$. Set $x, y, z \in 2 \mathbb{Z}$ such that $x<y<z$. Then we have $\left(h^{j+1}(y, 0)(1)-h^{j+1}(x, 0)(1), h^{j+1}(z, 0)(1)-\right.$ $\left.h^{j+1}(y, 0)(1)\right)=\left(h^{j}(y, 0)(1)-h^{j}(x, 0)(1), h^{j}(z, 0)(1)-h^{j}(y, 0)(1)\right)+\left(b_{\left(h^{j}(y, 0)\right)}-\right.$ $\left.b_{\left(h^{j}(x, 0)\right)}, b_{\left(h^{j}(z, 0)\right)}-b_{\left(h^{j}(y, 0)\right)}\right)$. By the independence of the family of random variables $\left\{b_{(x, t)}:(x, t) \in \mathbb{Z}_{\text {even }}^{2}\right\}$, it is clear that

$$
\begin{aligned}
& \left\{X_{j}=\left(\pi^{(y, 0)}(j)-\pi^{(x, 0)}(j), \pi^{(z, 0)}(j)-\pi^{(y, 0)}(j)\right)\right. \\
& \left.\quad=\left(h^{j}(y, 0)(1)-h^{j}(x, 0)(1), h^{j}(z, 0)(1)-h^{j}(y, 0)(1)\right): j \geq 0\right\}
\end{aligned}
$$

is a Markov chain with state space $\left\{(x, t) \in \mathbb{Z}^{2}: x, t \geq 0\right\}$, which verifies Condition (i) of Proposition 3.2

Finally, to verify Condition (ii), we take $\mathcal{S}_{1}=\emptyset$ so that part (b) of Condition (ii) is trivially satisfied. For Condition (a) of Proposition 3.2 (ii), let us denote, $b_{(x, 0)}=I_{x}, b_{(y, 0)}=I_{y}$ and $b_{(z, 0)}=I_{z}$. Hence,

$$
\begin{aligned}
\mathbb{E}[ & \left.X_{1}(1) X_{1}(2)-X_{0}(1) X_{0}(2) \mid X_{0}=(y-x, z-y)\right] \\
= & \mathbb{E}\left[\left(y-x+\left(I_{y}-I_{x}\right)\right)\left(z-y+\left(I_{z}-I_{y}\right)\right)\right. \\
& \left.\quad-(y-x)(z-y) \mid X_{0}=(y-x, z-y)\right] \\
= & \mathbb{E}\left[\left(y-x+\left(I_{y}-I_{x}\right)\right)\left(z-y+\left(I_{z}-I_{y}\right)\right)-(y-x)(z-y)\right] \\
= & \left.(z-y) \mathbb{E}\left(I_{y}-I_{x}\right)\right)+(y-x) \mathbb{E}\left(I_{z}-I_{y}\right)+\mathbb{E}\left[\left(I_{y}-I_{x}\right)\left(I_{z}-I_{y}\right)\right] .
\end{aligned}
$$

Clearly from definition, we have $\mathbb{E}\left(I_{x}\right)=\mathbb{E}\left(I_{y}\right)=\mathbb{E}\left(I_{z}\right)=0$ and $\mathbb{E}\left(I_{y}^{2}\right)=1$. Further for $x<y<z$, the random variables $I_{x}, I_{y}$ and $I_{z}$ are independent. Hence,

$$
\mathbb{E}\left[X_{1}(1) X_{1}(2)-X_{0}(1) X_{0}(2) \mid X_{0}=(y-x, z-y)\right]=-1 .
$$

If $x=y$ or $y=z$, i.e., at least one pair of paths have already coalesced, we have that $X_{1}(1) X_{1}(2)=0$. Therefore, $\mathcal{X}$ satisfies the conditions in Proposition 3.2 with the choice of $\mathcal{S}_{1}=\emptyset, d_{1}=-1$. This allows to conclude that Condition (B2) of Theorem 2.2 holds for $\left\{\overline{\mathcal{X}}_{n}: n \in \mathbb{N}\right\}$.

\subsection{Howard's model}

We first describe a 2-dimensional drainage network proposed by Howard (see [14]). Fix $0<p<1$ and let $\left\{B_{(x, t)}:(x, t) \in \mathbb{Z}^{2}\right\}$ be an i.i.d. collection of Bernoulli random variables with success probability $p$. Set $\mathcal{V}=\{(x, t) \in$ $\left.\mathbb{Z}^{2}: B_{(x, t)}=1\right\}$. Let $\left\{U_{(x, t)}:(x, t) \in \mathbb{Z}^{2}\right\}$ be an i.i.d. collection of Rademacher 
random variables, independent of the collection $\left\{B_{(x, t)}:(x, t) \in \mathbb{Z}^{2}\right\}: \mathbb{P}\left(U_{(x, t)}=\right.$ $1)=\mathbb{P}\left(U_{(x, t)}=-1\right)=1 / 2$. For a vertex $(x, t) \in \mathbb{Z}^{2}$, we consider $k_{0}=\min \{|k|$ : $\left.k \in \mathbb{Z}, B_{(x+k, t+1)}=1\right\}$. Clearly, $k_{0}$ is almost surely finite. Now, we define,

$$
h(x, t):= \begin{cases}\left(x+k_{0}, t+1\right) & \text { if }\left(x-k_{0}, t+1\right) \notin \mathcal{V} \\ \left(x-k_{0}, t+1\right) & \text { if }\left(x+k_{0}, t+1\right) \notin \mathcal{V} \\ \left(x+U_{(x, t)} k_{0}, t+1\right) & \text { otherwise. }\end{cases}
$$

Here each open vertex $(x, t) \in \mathcal{V}$ represents a water source and the edge $\langle(x, t), h(x, t)\rangle$ represents the channel through which water can flow. Consider the random graph $G=(\mathcal{V}, E)$ where the random edge set $E$ is given by $E=\{\langle(x, t), h(x, t)\rangle$ : $(x, t) \in \mathcal{V}\}$. Gangopadhyay et al. 13 . proved that this random graph $G$ is connected almost surely. This result, shows that the Howard's model generates a random directed tree on $\mathbb{Z}^{2}$. The authors of [13] actually used a general construction and studied this model for higher dimensions also. The construction that we presented here is applicable for $d=2$ only and agrees with that of 13 for $d=2$.

Let $\mathcal{X}$ be the collection of all paths obtained by following the edges and for $\gamma, \sigma>0$, let $\mathcal{X}_{n}=\mathcal{X}_{n}(\gamma, \sigma)$ be the collection of scaled paths with normalization constants $\gamma, \sigma$. Coletti et al. [5, 6] proved that, for $\gamma=1$ and $\sigma=\sigma_{0}(p)$ with $\sigma_{0}(p)$ as given by $\sigma_{0}(p)=\left(\frac{(1-p)\left(2-2 p+p^{2}\right)}{p^{2}(2-p)^{2}}\right)^{1 / 2}, \overline{\mathcal{X}}_{n}$ converges in distribution to the Brownian web. Again, to verify Condition $(B 2)$ they proved a partial FKG inequality for the Howard's model. We show here that Howard's model satisfies Proposition 3.2, and thereby Condition (B2) is verified.

By the i.i.d. nature of the collections of random variables $\left\{B_{(x, t)}:(x, t) \in\right.$ $\left.\mathbb{Z}^{2}\right\},\left\{U_{(x, t)}:(x, t) \in \mathbb{Z}^{2}\right\}$, it follows that $\mathcal{X}$ satisfies Condition (ii) of Proposition 3.2 It is not difficult to see that $\mathcal{X}$ consists of non-crossing paths only and Condition (i) of Proposition 3.2 holds. From definition it follows that for any $(y, s) \in \mathbb{Z}^{2}, h(y, s)$ depends only on the random variables $\left\{B_{(x, s+1)}: x \in \mathbb{Z}\right\}$ and $U_{(y, s)}$. Thus from the i.i.d. nature of the random variables it follows that for $x, y, z \in \mathbb{Z}$ with $x<y<z$, the process

$$
\begin{aligned}
& \left\{X_{j}=\left(\pi^{(y, 0)}(j)-\pi^{(x, 0)}(j), \pi^{(z, 0)}(j)-\pi^{(y, 0)}(j)\right)\right. \\
& \left.\quad=\left(h^{j}(y, 0)(1)-h^{j}(x, 0)(1), h^{j}(z, 0)(1)-h^{j}(y, 0)(1)\right): j \geq 0\right\}
\end{aligned}
$$

is a Markov chain with state space $\left\{(x, t) \in \mathbb{Z}^{2}: x, t \geq 0\right\}$, which verifies Condition (i) of Proposition 3.2

To verify Condition (ii), we take $\mathcal{S}_{1}=\left\{(l, m) \in \mathbb{Z}^{2}: 1 \leq \min (l, m) \leq r_{0}\right\}$ where $r_{0} \in \mathbb{N}$ will be chosen later. We observe that, for any $r_{0} \geq 1$,

$$
\mathbb{P}\left[X_{1} \in \mathcal{S}_{0} \mid X_{0}=(y-x, z-y)\right] \geq(1-p)^{2 r_{0}} p
$$

for all $((y-x),(z-y)) \in \mathcal{S}_{1}$. For this assume that $y-x \leq r_{0}$ and consider the event $\left\{B_{(u, 1)}=0\right.$, for $\left.|u-x| \leq r_{0}, u \neq y, B_{(y, 1)}=1\right\}$, i.e., the vertex above the second path is open and all other points closed which are within a distance $r_{0}$ (on both sides) from the vertex above the first path. This ensures that the first path and the second path coalesce. Similar arguments hold when $z-y \leq r_{0}$. This verifies part (b) of Condition (ii) Proposition 3.2 with $p_{0}=(1-p)^{2 r_{0}} p>0$. 
For part (a) of Condition (ii) in Proposition 3.2 , let us denote, $I_{x}:=h(x, 0)(1)-$ $x, I_{y}:=h(y, 0)(1)-y$ and $I_{z}:=h(z, 0)(1)-z$. As in the earlier model, we obtain,

$$
\begin{aligned}
& \mathbb{E}\left[X_{1}(1) X_{1}(2)-X_{0}(1) X_{0}(2) \mid X_{0}=(y-x, z-y)\right] \\
& =(z-y) \mathbb{E}\left(I_{y}-I_{x}\right)+(y-x) \mathbb{E}\left(I_{z}-I_{y}\right)+\mathbb{E}\left[\left(I_{y}-I_{x}\right)\left(I_{z}-I_{y}\right)\right] .
\end{aligned}
$$

Now, we use the fact that the marginal distributions of $I_{x}, I_{y}$ and $I_{z}$ are same as $I$ (say) with $\mathbb{E}(I)=0$. But as opposed to the earlier model, these random variables are no longer independent. Using Cauchy Schwartz inequality for any $x, y, z \in \mathbb{Z}$ with $x<y<z$ we have

$$
\begin{aligned}
& \mathbb{E}\left[X_{1}(1) X_{1}(2)-X_{0}(1) X_{0}(2) \mid X_{0}=(y-x, z-y)\right] \\
& =\mathbb{E}\left[\left(I_{y}-I_{x}\right)\left(I_{z}-I_{y}\right)\right] \leq 4 \mathbb{E}\left[I^{2}\right]<\infty .
\end{aligned}
$$

To verify part (a) of the Condition (ii) of Proposition 3.2 we still need to show that for $((y-x),(z-y)) \in\left(\mathcal{S}_{0} \cup \mathcal{S}_{1}\right)^{c}$ there exists $d_{1}>0$ such that $\mathbb{E}\left[X_{1}(1) X_{1}(2)-X_{0}(1) X_{0}(2) \mid X_{0}=(y-x, z-y)\right] \leq-d_{1}$. For $x<y<z \in \mathbb{Z}$ with $\min (y-x, z-y)>r>0$ we have

$$
\begin{aligned}
& \mathbb{E}\left[X_{1}(1) X_{1}(2)-X_{0}(1) X_{0}(2) \mid X_{0}=(y-x, z-y)\right] \\
& =\mathbb{E}\left[\left(I_{y}-I_{x}\right)\left(I_{z}-I_{y}\right)\right]=-\mathbb{E}\left[I_{y}^{2}\right]+\mathbb{E}\left[I_{y} I_{z}\right]-\mathbb{E}\left[I_{x} I_{z}\right]+\mathbb{E}\left[I_{x} I_{y}\right] .
\end{aligned}
$$

The last three terms converge to 0 as $r \rightarrow \infty$. We show it for $\mathbb{E}\left[I_{y} I_{z}\right]$, the others being exactly the same. Observe that $\mathbb{E}\left[\left(I_{y} I_{z}\right) \mathbb{I}\left(\max \left\{\left|I_{y}\right|,\left|I_{z}\right|\right\}<r / 2\right)\right]=0$ and hence we obtain

$$
\begin{aligned}
& \left.\mid \mathbb{E}\left[I_{y} I_{z}\right)\right] \mid \\
& =\left|\mathbb{E}\left[\left(I_{y} I_{z}\right) \mathbb{I}\left(\max \left\{\left|I_{y}\right|,\left|I_{z}\right|\right\}<r / 2\right)\right]+\mathbb{E}\left[\left(I_{y} I_{z}\right) \mathbb{I}\left(\max \left\{\left|I_{y}\right|,\left|I_{z}\right|\right\} \geq r / 2\right)\right]\right| \\
& \leq \sqrt{\mathbb{E}\left[I_{y}^{2} I_{z}^{2}\right]} \sqrt{\mathbb{P}\left(\max \left\{\left|I_{y}\right|,\left|I_{z}\right|\right\} \geq r / 2\right)} \\
& \leq \sqrt[4]{\mathbb{E}\left[I_{y}^{4}\right]} \sqrt[4]{\mathbb{E}\left[I_{z}^{4}\right]} \sqrt{2} \sqrt{\mathbb{P}\left(\left|I_{y}\right|>r / 2\right)} \\
& \rightarrow 0
\end{aligned}
$$

as $r \rightarrow \infty$. Thus, we have $-\mathbb{E}\left[I_{y}^{2}\right]+\mathbb{E}\left[I_{y} I_{z}\right]-\mathbb{E}\left[I_{x} I_{z}\right]+\mathbb{E}\left[I_{x} I_{y}\right] \leq-\mathbb{E}\left(I^{2}\right) / 2$ for all $r>r_{0}$. Hence it follows that Howard's model satisfies Proposition 3.2 with $\mathcal{S}_{1}=\left\{(l, m) \in \mathbb{Z}^{2}: 1 \leq \min \{l, m\} \leq r_{0}\right\}, d_{1}=\mathbb{E}\left(I^{2}\right) / 2, d_{2}=4 \mathbb{E}\left[I^{2}\right]$ and $p_{0}=(1-p)^{2 r_{0}} p$ and Condition (ii) of Proposition 2.3 holds for this discrete model. This shows that the condition (B2) is satisfied.

\subsection{Ferrari, Landim and Thorisson's model}

We are interested in a directed forest spanning on Poisson process on $\mathbb{R}^{2}$ introduced by Ferrari et al. 11. The vertices of the forest are the atoms of a homogeneous Poisson point process $N$ having unit intensity in $\mathbb{R}^{2}$, embedded with the Euclidean norm. With an abuse of notation, the Poisson point process $N$ will be considered in the sequel sometimes as a point measure with Dirac masses on its atoms and sometimes as a union of points of $\mathbb{R}^{2}$. The ancestor of $(x, t) \in \mathbb{R}^{2}$, denoted by $h(x, t)$, is the closest point $\left(x^{\prime}, t^{\prime}\right) \in N$ such that

$$
t^{\prime}>t \quad \text { and } \quad\left|x^{\prime}-x\right|<\frac{1}{2} \text {. }
$$


This means that in order to find the ancestor of $(x, t)$, we are growing a tube of width 1 in the upward direction whose base is centered around $x$. The first point of $N$ encountered by the tube is the ancestor of $(x, t)$.

The graph, considered by Ferrari et al. [11, with vertices as the atoms of $N$ and edge set $\{\langle(x, t), h(x, t)\rangle,(x, t) \in N\}$, is of out-degree 1 almost surely and hence a forest. We should mention here that the authors of [11] considered this forest on $\mathbb{R}^{d}$ for general $d$ and showed that for $d=2$, the random forest is indeed a tree, i.e., connected almost surely. We concentrate only on $d=2$. In another work, Ferrari, Fontes and Wu [12] showed that for $d=2$ under diffusive scaling, as a collection of paths, this tree converges to the Brownian web.

It is not apriori clear that Condition (iii) of Proposition 2.3 holds in this model. However, we show that the main idea of Proposition 2.3 can be extended to give an alternate verification of (B2) for this model as well.

For any path $\pi \in \mathcal{X}$ emanating from $(x, t)$, we define a càdlàg path, denoted by $\tilde{\pi}$ henceforth, emanating from $(x, t)$ to its ancestor $h(x, t)=\left(x^{\prime}, t^{\prime}\right)$ which remains constant on $\left[t, t^{\prime}\right)$ with value $x$ and then jumps to $x^{\prime}$. It is easy to observe that $\pi$ and $\tilde{\pi}$ meet at jump times of $\tilde{\pi}$ and hence the number of steps between two time segments are exactly the same for both types of paths. From now on, we will only consider the càdlàg paths.

In the sequel, we will deal with triplets $(x, y, z) \in \mathbb{R}^{3}$ with $x<y<$ $z$ and $\min \{(y-x),(z-y)\} \geq 1 / 2$. Let $X_{t}=\left(\tilde{\pi}^{(x, 0)}(t), \tilde{\pi}^{(y, 0)}(t), \tilde{\pi}^{(z, 0)}(t)\right)$ denote the triplet of càdlàg paths starting from the points $(x, 0),(y, 0)$ and $(z, 0)$ respectively. Further, it is useful to observe that our choice of starting points ensures that either $\min \left\{\left(X_{t}(2)-X_{t}(1)\right),\left(X_{t}(3)-X_{t}(2)\right)\right\} \geq 1 / 2$ or $\left(X_{t}(2)-X_{t}(1)\right)\left(X_{t}(3)-X_{t}(2)\right)=0$ for all $t \geq 0$ where $X_{t}(i)$ denotes the $i$-th coordinate of $X_{t}$. In other words, the paths are separated by distance $1 / 2$ or they have coalesced for all time points. Hence, the process $\left\{X_{t}: t \geq 0\right\}$, is a pure jump Markov process with state space $\mathcal{M}=\{(u, v, w): u \leq v \leq w$, either $u=$ $v$ or $v=w$ or $\min \{v-u, w-v\} \geq 1 / 2\}$.

This part of the argument is motivated from Ferrarri et. al. 12. We are interested in finding $\lim _{n \rightarrow \infty} \mathbb{P}\left(\eta_{\mathcal{X}}(0, n ; 0, \epsilon \sqrt{n}) \geq 3\right)$. Set $A_{n}$ as the (random) point set $\left\{\tilde{\pi}(0): \pi \in \mathcal{X}, \sigma_{\pi} \leq 0\right\} \cap[-1 / 2, \epsilon \sqrt{n}+1 / 2]$. It is easy to observe that for any $x, y \in A_{n}$ we must have $|x-y| \geq 1 / 2$. Hence elements of the set $A_{n}$ can be enumerated as $\left\{x_{1}, x_{2}, \ldots, x_{J}\right\}$ where $x_{1}, \ldots, x_{J}$ and $J$ are random variables such that $x_{1}<x_{2}<\cdots<x_{J}$ and $x_{i+1}-x_{i} \geq 1 / 2$ almost surely for $1 \leq i \leq J-1$ (with convention that if $J=0$, the point set is empty). If required, we consider an enlarged set $B_{n}$ containing $A_{n}$ such that $B_{n}=\left\{y_{1}, y_{2}, \ldots, y_{J^{\prime}}\right\}$ where

$$
y_{1} \geq-1 / 2, y_{J^{\prime}} \leq \epsilon \sqrt{n}+1 / 2 \text { and } 1 / 2 \leq y_{i+1}-y_{i} \leq 1 \text { for } 1 \leq i \leq J^{\prime}-1 .
$$

Note that $J^{\prime}$ is random but that we necessarily have $J^{\prime} \leq 2 \epsilon \sqrt{n}+2$.

For $1 \leq j \leq J^{\prime}-2$, let us define the event

$$
E(j)=\left\{\tilde{\pi}^{\left(y_{j}, 0\right)}(n)<\tilde{\pi}^{\left(y_{j+1}, 0\right)}(n)<\tilde{\pi}^{\left(y_{J^{\prime}}, 0\right)}(n)\right\} .
$$

On the event $\left\{\eta_{\mathcal{X}}(0, n ; 0, \epsilon \sqrt{n}) \geq 3\right\}$, there must exist $j=j(\omega) \in\left\{1,2, \cdots, J^{\prime}-\right.$ $2\}$ such that $E(j)$ is satisfied. Let $\mathcal{F}_{0}$ denote the $\sigma$-field generated by the Poisson point process on the negative half-plane $\left\{(x, t) \in \mathbb{R}^{2}: t \leq 0\right\}$. Clearly, $A_{n}$ is $\mathcal{F}_{0}$ measurable. Given the set $A_{n}$, the set $B_{n}$ can be chosen by a deterministic algorithm so that $B_{n}$, and hence $J^{\prime}$, are $\mathcal{F}_{0}$ measurable. On the other hand, 
given $B_{n}$, the event $E(j)$ depends only on Poisson process on the upper halfplane $\left\{(x, t) \in \mathbb{R}^{2}: t>0\right\}$ and hence $E(j)$ depends on $\mathcal{F}_{0}$ only through the positions of $B_{n}$.

We prove the following proposition.

Proposition 4.1. For any set $B_{n}$ having properties as above, we have, for $j \geq 1$,

$$
\mathbb{P}\left(E(j) \mid B_{n}\right) \leq \frac{1}{n}\left[C_{1}+C_{2} \epsilon \sqrt{n}\right]
$$

for constants $C_{1}, C_{2}>0$ independent of $j$, as well as $B_{n}$.

Assuming the Proposition 4.1, we have

$$
\begin{aligned}
& \mathbb{P}\left(\eta_{\mathcal{X}}(0, n ; 0, \epsilon \sqrt{n}) \geq 3\right)=\mathbb{E}\left(\mathbb{P}\left(\left(\eta_{\mathcal{X}}(0, n ; 0, \epsilon \sqrt{n}) \geq 3\right) \mid \mathcal{F}_{0}\right)\right) \\
& \leq \mathbb{E}\left(\mathbb{P}\left(\cup_{j=1}^{J^{\prime}-2} E(j) \mid \mathcal{F}_{0}\right)\right) \leq \mathbb{E}\left(\sum_{j=1}^{J^{\prime}-2} \mathbb{P}\left(E(j) \mid \mathcal{F}_{0}\right)\right) \\
& =\mathbb{E}\left(\sum_{j=1}^{J^{\prime}-2} \mathbb{P}\left(E(j) \mid B_{n}\right)\right) \leq \mathbb{E}\left(\sum_{j=1}^{J^{\prime}-2} \frac{1}{n}\left[C_{1}+C_{2} \epsilon \sqrt{n}\right]\right) \\
& =\frac{1}{n}\left[C_{1}+C_{2} \epsilon \sqrt{n}\right] \mathbb{E}\left(J^{\prime}-2\right) \leq \frac{1}{n}\left[C_{1}+C_{2} \epsilon \sqrt{n}\right] 2 \epsilon \sqrt{n} \\
& \rightarrow 2 C_{2} \epsilon^{2}
\end{aligned}
$$

as $n \rightarrow \infty$, which proves (B2).

\subsubsection{A Lyapunov condition for càdlàg branches}

It now remains to prove Proposition 4.1. We are inspired by a result due to Meyn and Tweedie [16, Theorem 4.3], which is based on establishing a Lyapunov control similar to Theorem 3.1(i) on the infinitesimal generator of $\left(X_{t}\right)_{t \geq 0}$ (see e.g. 9] or [16] for definitions of these generators).

Let us define $\mathcal{M}_{0}=\{(u, v, w): u \leq v \leq w$, either $u=v$ or $v=w\}$ and $\tau=\tau\left(\mathcal{M}_{0}\right)=\inf \left\{t \geq 0, X_{t} \in \mathcal{M}_{0}\right\}$. We note that $\tau<\infty$ almost surely.

Theorem 4.2. Assume that there exist $d>0$ and a non-negative function $V: \mathcal{M} \rightarrow[0, \infty)$ such that the generator $G$ of the Markov process $\left(X_{t}\right)_{t \geq 0}$ satisfies

$$
G V(u, v, w) \leq-d \mathbf{1}_{\left(\mathcal{M}_{0}\right)^{c}}(u, v, w),
$$

Then, for any $(u, v, w) \in\left(\mathcal{M}_{0}\right)^{c}$,

$$
\mathbb{E}_{(u, v, w)}(\tau) \leq \frac{1}{d} V(u, v, w) .
$$

Theorem 4.2. The result is a particular case of [16] (Th. 4.3). Using Dynkin's formula:

$$
\begin{aligned}
0 & \leq \mathbb{E}_{(u, v, w)}\left(V\left(X_{\tau \wedge t}\right)\right) \\
& \leq V(u, v, w)+\mathbb{E}_{(u, v, w)}\left(\int_{0}^{\tau \wedge t}\left(-d \mathbf{1}_{\left(\mathcal{M}_{0}\right)^{c}}\left(X_{s}\right)\right) d s\right) \\
& \leq V(u, v, w)-d \mathbb{E}_{(u, v, w)}(\tau \wedge t)
\end{aligned}
$$

from which we obtain the announced result by letting $t \rightarrow \infty$. 
We claim that for the function

$$
V(u, v, w):=(v-u)(w-v)
$$

the equation (22) is satisfied with $d=1 / 12$. Assuming the claim, we first prove Proposition 4.1

Proposition 4.1. With $\tau$ being as defined before Theorem4.2 for $j \in\left\{1, \ldots, J^{\prime}-\right.$ $2\}$,

$$
\begin{aligned}
\mathbb{P}\left(E(j) \mid B_{n}\right) & =\mathbb{P}_{\left(y_{j}, y_{j+1}, y_{J^{\prime}}\right)}(\tau>n) \leq \frac{1}{n} \mathbb{E}_{\left(y_{j}, y_{j+1}, y_{J^{\prime}}\right)}(\tau) \\
& \leq \frac{12}{n}\left(y_{j+1}-y_{j}\right)\left(y_{J^{\prime}}-y_{j+1}\right) \leq \frac{12}{n}[\epsilon \sqrt{n}+1]
\end{aligned}
$$

using the fact that $y_{j+1}-y_{j} \leq 1$ and $y_{J^{\prime}}-y_{j+1} \leq \epsilon \sqrt{n}+1$.

Now we verify the claim above. For this, we start with computing the generator of $\left(X_{t}\right)_{t>0}$. The basic idea is the following: the generator computed on $V$ can be interpreted as an expectation, which gives us a way to estimate it easily. Let us fix $(u, v, w) \in\left(\mathcal{M}_{0}\right)^{c}$. We need some notation. Let $\mathcal{J}=[-1 / 2,1 / 2]$ and $A=(u+\mathcal{J}) \cup(v+\mathcal{J}) \cup(w+\mathcal{J})$. Note that these sets in $A$ may overlap and the total length (Lebesgue measure) $|A|$ of $A$ can be strictly less than 3 . Consider a uniform random variable $U$ on the set $A$. Now, for $s \in\{u, v, w\}$, define $I_{s}$ as follows:

$$
I_{s}:= \begin{cases}U-s & \text { if } U \in s+\mathcal{J} \\ 0 & \text { otherwise. }\end{cases}
$$

Note that $I_{s}$ denotes the increment of the jump of the path starting from $(s, 0)$ at the first jump time of the process $\left(X_{t}\right)_{t \geq 0}$. Then we have

$$
G V(u, v, w)=|A| \mathbb{E}\left[V\left(u+I_{u}, v+I_{v}, w+I_{w}\right)-V(u, v, w)\right] .
$$

Using the form of $V$, we have

$$
G V(u, v, w)=|A| \mathbb{E}\left[(v-u)\left(I_{w}-I_{v}\right)+(w-v)\left(I_{v}-I_{u}\right)+\left(I_{w}-I_{v}\right)\left(I_{v}-I_{u}\right)\right] .
$$

First we make a few observations on $I_{s}$ for $s \in\{u, v, w\}$. Clearly, $I_{s}$ is symmetric and hence $\mathbb{E}\left(I_{s}\right)=0$. Further, $I_{u} I_{w}=0$ almost surely since $(u+$ $\mathcal{J}) \cap(w+\mathcal{J})=\emptyset$ as $(u, v, w) \in\left(\mathcal{M}_{0}\right)^{c}$. Also, we observe that $I_{u} I_{v}$ is non-zero only when $U \in(u+\mathcal{J}) \cap(v+\mathcal{J})$. In such a case, $I_{u}>0$ and $I_{v}<0$. Thus, $I_{u} I_{v} \leq 0$ almost surely. Similar argument shows that $I_{v} I_{w} \leq 0$ almost surely. Now,

$$
\mathbb{E}\left(I_{v}^{2}\right)=\frac{1}{|A|} \int_{-1 / 2}^{1 / 2} t^{2} d t=\frac{1}{12|A|} .
$$

Thus, putting back in (24), we have

$$
G V(u, v, w)=|A| \mathbb{E}\left[\left(I_{w}-I_{v}\right)\left(I_{v}-I_{u}\right)\right] \leq-|A| \mathbb{E}\left(I_{v}^{2}\right)=-\frac{1}{12} .
$$

This proves the claim that $V$ satisfies the condition (22) with $d=1 / 12$. 


\section{Acknowledgement}

The authors thank Rongfeng Sun for careful reading and valuable comments. This work was supported by the GdR GeoSto 3477 and Labex CEMPI (ANR11-LABX-0007-01). D.C. is funded by ANR PPPP (ANR-16-CE40-0016). A.S. wishes to thank Science \& Engineering Research Board (SERB), Department of Science \& Technology for the grant (MTR/2017/000293). This work was partially done while K.S., A.S. and V.C.T. were visiting the Institute for Mathematical Sciences, National University of Singapore in 2017. The visit was supported by the Institute.

\section{References}

[1] R. Arratia, "Coalescing Brownian motions on the line", Ph.D. thesis, University of Wisconsin, Madison, 1979.

[2] F. Baccelli and C. Bordenave, "The radial spanning tree of a Poisson point process" Ann. Appl. Probab., 17(1): 305-359, 2007.

[3] N. Berestycki, C. Garban and A. Sen, "Coalescing Brownian flows: a new approach". Ann. Probab., 43(6): 3177-3215, 2015.

[4] P. Billingsley, "Probability and measure", Second Edition., John Wiley and Sons, 1986.

[5] C.-F. Coletti, E. S. Dias and L. R. G. Fontes, "Scaling limit for a drainage network model", J. Appl. Probab., 46, 1184-1197, 2009.

[6] C. Coletti and G. Valle, "Convergence to the Brownian web for a generalization of the drainage network model", Ann. Inst. H. Poincaré Probab. Statist., 50:(3), 899-919, 2014.

[7] D. Coupier, K. Saha, A. Sarkar, V.C. Tran, "The 2d-directed spanning forest converges to the Brownian web", arXiv:1805.09399, 2018.

[8] D. Coupier, V.C. Tran, "The 2d-directed spanning forest is almost surely a tree", Random Structures and Algorithms, 42(1), 59-72, 2013.

[9] S.N. Ethier and T.G. Kurtz, "Markov Processes: Characterization and Convergence", Wiley, 2009.

[10] L. R. G. Fontes, M. Isopi, C. M. Newman and K. Ravishankar, "The Brownian web: characterization and convergence", Ann. Probab., 32, 2857-2883, 2004.

[11] P. Ferrari, C. Landim and H. Thorisson, "Poisson trees, succession lines and coalescing random walks", Ann. Inst. H. Poincaré Probab. Statist., 40(2), 141-152, 2004.

[12] P. A. Ferrari, L. R. G. Fontes and X. Y. Wu, "Two-dimensional Poisson Trees converge to the Brownian web", Ann. Inst. H. Poincaré Probab. Statist., 41, 851-858, 2005. 
[13] S. Gangopadhyay, R. Roy and A. Sarkar, "Random oriented trees: a model of drainage networks", Ann. Appl. Probab., 14, 1242-1266, 2004.

[14] A. D. Howard, "Simulation of stream networks by headward growth and branching", Geogr. Anal., 3, 29-50, 1971.

[15] S. P. Meyn and R. L. Tweedie, "Markov chains and stochastic stability", Springer-Verlag, London, 1993.

[16] S. P. Meyn and R. L. Tweedie, "Stability of Markovian processes III: Foster-Lyapunov criteria for continuous-time processes", Advances in Applied Probability, 25(3), 518-548, 1993.

[17] C. M. Newman, K. Ravishankar and R. Sun, "Convergence of coalescing nonsimple random walks to the Brownian web", Electron. J. Prob., 10, 21-60, 2005.

[18] I. Rodriguez-Iturbe and A. Rinaldo, "Fractal river basins: chance and self-organization", Cambridge University Press, New York (1997).

[19] R. Roy, K. Saha, and A. Sarkar, "Random directed forest and the Brownian web", Ann. Inst. H. Poincaré Probab. Statist., 52, 1106-1143., 2005.

[20] R. Roy, K. Saha, and A. Sarkar, "Hack's law in a drainage network model: a Brownian web approach", Ann Appl. Probab., 26:3, 1807-1836, 2016.

[21] A. Sarkar and R. Sun, "Brownian web in the scaling limit of supercritical oriented percolation in dimension $1+1$ ", Electron. J. of Probab., 18, $1-23,2013$.

[22] A. E. Scheidegger, "A stochastic model for drainage pattern into an intramontane trench", Bull. Ass. Sci. Hydrol., 12, 15-20, 1967.

[23] E. Schertzer, R. Sun, and J. Swart, "The Brownian web, the Brownian net, and their universality", Advances in Disordered Systems, Random Processes and Some Applications, 270-368, Cambridge University Press, 2017.

[24] A. Sturm and J.M. Swart, "A particle system with cooperative branching and coalescence", Ann. of Appl. Probab., 15:3, 1616-1649, 2015.

[25] R. Sun and J. Swart, "The Brownian net", Ann. Probab., 36, 1153-1208, 2008 .

[26] B. Tóth and W. Werner, "The true self-repelling motion", Probab. Theory Related Fields, 111, 375-452, 1998.

[27] G. Valle and L. Zuaznabar, "A version of the random directed forest and its convergence to the Brownian web", arXiv:1704.05555, 2017. 